$f(x)=$ const on $[0,1], A_{f}=1$. So here $A_{f} \geq L_{f}$; i.e., in conjunction with $3.1, A_{f}=L_{f}$. (It is also easy to show directly that $L_{f}=1$.) In conclusion, I would like to express my thanks to Dr. Peter Lax, who introduced me to this subject, and with whom I have had many helpful discussions.

\title{
REFERENCES
}

1. A. Beurling, $A$ theorem on functions defined on a semigroup, Math. Scand vol. 1 (1953) pp. 127-130.

2. L. Schwartz, Théorie générale des fonctions moyenne-périodiques, Ann. of Math. vol. 48 (1947) pp. 857-929.

3. J.-P. Kahane, Sur quelques problèmes d'unicité et de prolongement . . ., Annales de l'Institut Fourier vol. 5 (1953-1954) Ch. I, §1, pp. 41-48.

4. P. Koosis, Note sur les fonctions moyenne-périodiques, Annales de l'Institut Fourier vol. 6 (1955-1956) pp. 357-360.

5. R. Nevanlinna, Eindeutige analytische Funktionen, Berlin-Springer, 2d ed., 1953.

6. R. Boas, Entire functions, New York, Academic Press, 1954.

7. B. Nyman, On the one-dimensional translation group and semi-group in certain function spaces, thesis, Uppsala, 1950.

New YoRk UNIVERSITY

\section{FUNCTIONAL EQUATIONS IN THE THEORY OF DYNAMIC PROGRAMMING-VII. A PARTIAL DIFFERENTIAL EQUATION FOR THE FREDHOLM RESOLVENT}

\section{RICHARD BELLMAN}

1. Introduction. Let $K(x, y)$ be a symmetric kernel over the square $0 \leqq x, y \leqq T$, continuous in both variables in this region, and possessing the additional property that $\int_{0}^{T} \int_{0}^{T} K(x, y) u(x) u(y) d x d y+\int_{0}^{T} u^{2}(x) d x$ is positive definite. Then the Fredholm integral equation

$$
u(x)+v(x)+\int_{a}^{T} K(x, y) u(y) d y=0, \quad 0 \leqq a \leqq T,
$$

has a unique solution for any function $v(x)$ continuous for $a \leqq x \leqq T$. This solution may be represented in the form

$$
u(x)=-v(x)+\int_{a}^{T} Q(x, y, a) v(y) d y .
$$

Let us call the kernel $Q(x, y, a)$ the Fredholm resolvent.

The purpose of this note is to show that $Q(x, y, a)$ satisfies the

Received by the editors August 2, 1956 and, in revised form, September 24, 1956. 
Riccati-type partial differential equation

$$
\frac{\partial Q}{\partial a}(x, y, a)=Q(a, x, a) Q(a, y, a) .
$$

It seems likely that this relation will be of service in connection with the computational solution of linear integral equations, but we shall not discuss this here.

To derive the result, we shall employ the functional equation technique of the theory of dynamic programming, cf. $[1 ; 3] .{ }^{1}$

2. A quadratic functional. Consider the quadratic functional

$$
\begin{aligned}
J(u)= & \int_{a}^{T} u^{2}(x) d x+2 \int_{a}^{T} u(x) v(x) d x \\
& +\int_{a}^{T} \int_{a}^{T} K(x, y) u(x) u(y) d x d y,
\end{aligned}
$$

$0 \leqq a \leqq T$, where $K(x, y)$ and $v(x)$ satisfy the conditions described in the first section. The minimum of $J(u)$ over all functions $u(x)$ which are continuous in $a \leqq x \leqq T$ exists and is attained by the solution of the Fredholm integral equation

$$
u(x)+v(x)+\int_{a}^{T} K(x, y) u(y) d y=0 .
$$

Utilizing the representation for $u(x)$ given in (1.2), we have

$$
\begin{aligned}
\underset{u}{\operatorname{Min} J(u)=} & \int_{a}^{T}\left(u(x)+v(x)+\int_{a}^{T} K(x, y) u(y) d y\right) u(x) d x \\
& +\int_{a}^{T} u(x) v(x) d x \\
= & -\int_{a}^{T} v^{2}(x) d x+\int_{a}^{T} \int_{a}^{T} Q(x, y, a) v(x) v(y) d x d y .
\end{aligned}
$$

Let us call this new functional, which depends upon $v(x)$ and $a, f(v(x), a)$. Thus

$$
f(v(x), a)=\operatorname{Min}_{u} J(u) .
$$

3. Functional equations. Using the fact that $f(v(x), a)$ is defined as a

1 We have in the meantime, using a different method, obtained the generalization of this result valid for kernels which are not necessarily symmetric. (Added in proof, May, 1957). 
minimum of a functional, let us employ the functional equation technique of dynamic programming to derive a functional equation for $f(v(x), a)$. This technique, as applied to integral equations, was sketched in [3].

Let us write, for $a<a+s<T$,

$$
\begin{aligned}
J(u)= & \int_{a}^{a+s} u^{2}(x) d x+2 \int_{a}^{a+s} u(x) v(x) d x \\
& +2 \int_{a}^{a+s} \int_{a+s}^{T} K(x, y) u(x) u(y) d x d y \\
& +\int_{a+s}^{T} u^{2}(x) d x+2 \int_{a+s}^{T} u(x) v(x) d x \\
& +\int_{a+s}^{T} \int_{a+s}^{T} K(x, y) u(x) u(y) d x d y \\
& +\int_{a}^{a+s} \int_{a}^{a+s} K(x, y) u(x) u(y) d x d y .
\end{aligned}
$$

Let $u(x)$ be the extremal function, which we know to be continuous as a function of $x$ in $a \leqq x \leqq T$, as a function of $a$ for $0 \leqq a \leqq T$, and as a function of $v(x)$. For small $s$, we may write, using the mean value theorem,

$$
\begin{aligned}
J(u)=s & {\left[u^{2}(a)+2 u(a) v(a)+2 u(a) \int_{a}^{T} K(a, y) u(y) d y\right] } \\
& +\int_{a+s}^{T} u^{2}(x) d x+2 \int_{a+s}^{T} u(x) v(x) d x \\
& +\int_{a+s}^{T} \int_{a+s}^{T} K(x, y) u(x) u(y) d x d y+o(s),
\end{aligned}
$$

where the remainder term is uniform for $|v(x)| \leqq m_{1}, 0 \leqq x \leqq T$, $0 \leqq a \leqq T$.

Let us rewrite this

$$
\begin{aligned}
J(u)= & s\left[u^{2}(a)+2 u(a) v(a)\right]+\int_{a+s}^{T} u^{2}(x) d x \\
& +2 \int_{a+\&}^{T} u(x)[v(x)+s u(a) K(a, x)] d x \\
& +\int_{a+s}^{T} \int_{a+\varepsilon}^{T} K(x, y) u(x) u(y) d x d y+o(s) .
\end{aligned}
$$


Employing the principle of optimality, we see that for $u(x)$, the extremal function, we must have

(4) $J(u)=s\left[u^{2}(a)+2 u(a) v(a)\right]+f(u(x)+s u(a) k(a, x), a+s)+o(s)$.

Hence

$$
\begin{aligned}
f(v, a)=\operatorname{Min}_{u} J(u) & =\underset{u(a)}{\operatorname{Min}}\left[s\left[u^{2}(a)+2 u(a) v(a)\right]\right. \\
& +f(u(x)+s u(a) K(a, x), a+s)]+o(s) .
\end{aligned}
$$

Since $f(v, a)$ is clearly a differentiable function of $v(x)$ and $a$, as we see upon referring to (3.3), we may obtain an integro-differential equation for $f$ upon letting $s \rightarrow+0$.

Let us define

$$
L(w(x), a)=\lim _{s \rightarrow 0} \frac{f(v(x)+s w(x), a)-f(v(x), a)}{s} .
$$

Then the limiting form of (5) is

$$
0=\operatorname{Min}_{u(a)}\left[u^{2}(a)+2 u(a) v(a)+u(a) L(K(a, x), a)+\frac{\partial f}{\partial a}\right] .
$$

The minimum is attained at

$$
u(a)=-v(a)-L(K(a, x), a) / 2,
$$

yielding the relation

$$
\frac{\partial f}{\partial a}=(v(a)+L(K(a, x), a) / 2)^{2} .
$$

4. The form of $L(w(x), a)$. Let us now compute $L(w(x), a)$. We have

$$
\begin{aligned}
f(v(x)+s w(x), a)= & -\int_{a}^{T} v^{2}(x) d x-2 s \int_{a}^{T} v(x) w(x) d x \\
& +\int_{a}^{T} \int_{a}^{T} Q(x, y, a) v(x) v(y) d x d y \\
& +2 s \int_{a}^{T} \int_{a}^{T} Q(x, y, a) v(x) w(x) d x d y+o(s) .
\end{aligned}
$$

Hence

$$
\begin{aligned}
& L(w(x), a) \\
& \quad=-2 \int_{a}^{T} v(x) w(x) d x+2 \int_{a}^{T} \int_{a}^{T} Q(x, y, a) v(x) w(y) d x d y .
\end{aligned}
$$


Thus

$$
\begin{aligned}
& L(K(a, x), a) \\
& =-2 \int_{a}^{T} v(x) K(a, x) d x+2 \int_{a}^{T} \int_{a}^{T} Q(x, y, a) v(x) K(a, y) d x d y .
\end{aligned}
$$

5. The functional equation for $Q(x, y, a)$. The equation of (3.9) then takes the form

$$
\begin{aligned}
\frac{\partial f}{\partial a}= & +v^{2}(a) \\
& +v(a)\left[-2 \int_{a}^{T} v(x) K(a, x) d x\right. \\
& +\frac{1}{4}\left[-2 \int_{a}^{T} v(x) K(a, x) d x\right. \\
= & \left.+2 \int_{a}^{T} \int_{a}^{T} Q(x, y, a) v(x) K(a, y) d x d y\right] \\
& \left.+\frac{1}{4} \int_{a}^{T} \int_{a}^{T} v(x, y, a) v(x) K(a, y) d x d y\right]^{2} \\
& \cdot 2 v(a)\left[\int_{a}^{T} v(x)\{-K(a, x)\right. \\
& {\left[-2 K\left(a, x_{2}\right)+2 \int_{a}^{T} Q\left(x_{2}, y_{2}, a\right) K\left(a, y_{2}\right) d y_{2}\right] d x_{1} d x_{2} . }
\end{aligned}
$$

(1)

On the other hand, using the expression for $f(v, a)$ given in (2.3), we have, carrying through the required differentiation

$$
\begin{aligned}
\frac{\partial f}{\partial a}= & +v^{2}(a)-2 v(a) \int_{a}^{T} Q(a, y, a) v(y) d y \\
& +\int_{a}^{T} \int_{a}^{T} \frac{\partial Q(x, y, a)}{\partial a} v(x) v(y) d x d y .
\end{aligned}
$$


Equating coefficients, we obtain the two relations

$$
Q(a, y, a)=K(a, y)-\int_{a}^{T} Q(y, z, a) K(a, z) d z
$$

and

$$
\begin{aligned}
\frac{\partial Q(a, y, a)}{\partial a}= & {\left[-K(a, x)+\int_{a}^{T} Q\left(x, z_{1}, a\right) K\left(a, z_{1}\right) d z_{1}\right] } \\
& \cdot\left[-K(a, y)+\int_{a}^{T} Q\left(y, z_{2}, a\right) K\left(a, z_{2}\right) d z_{2}\right]
\end{aligned}
$$

for $a \leqq x, y \leqq T$.

Note that (3) is readily derived, ab initio, by straightforward substitution of (1.2) in (1.1).

Combining (3) and (4), we obtain the result stated in (1.3). This completes the proof.

\section{BiBLIOGRAPHY}

1. R. Bellman, The theory of dynamic programming, Bull. Amer. Math. Soc., vol. 60 (1954) pp. 503-516.

2. - Functional equations in the theory of dynamic programming-I, Functions of points and point transformations, Trans. Amer. Math. Soc. vol. 80 (1955) pp. 55-71.

3. - Dynamic programming and a new formalism in the theory of integral equations, Proc. Nat. Acad. Sci. U.S.A. vol. 41 (1955) pp. 31-34.

The Rand Corporation 